\title{
AN ENERGY-BASED APPROACH TO ASSESS AND PREDICT EROSIVE AIRFOIL DEFOULING
}

\author{
ARTHUR RUDEK ${ }^{1,2}$, THOMAS-ALEXANDER ZITZMANN ${ }^{1}$, GERALD RUSS $^{1} \&$ BARRY DUIGNAN $^{2}$ \\ ${ }^{1}$ Hochschule Darmstadt, University of Applied Sciences, Germany. \\ ${ }^{2}$ Dublin Institute of Technology, Ireland.
}

\begin{abstract}
A dynamic indentation experiment is presented for assessment of the adhesive behavior of a range of coatings in erosive defouling of commercial aircraft engines using $\mathrm{CO}_{2}$ dry-ice. A series of experiments is presented in which particles made from a reference material (polyoxymethylene - POM) and from $\mathrm{CO}_{2}$ dry-ice are made to impact compressor airfoils under a range of impact angle and velocity conditions. The airfoils investigated are coated with an indicator material (PTFE), which is typically used to visualise the defouling effect in large scale compressor defouling experiments. In addition, fouled compressor airfoils taken from service and coated with a fouling typically found in low-pressure compressor stages are investigated. The energy required for the reference particles (POM) to create a defouling effect for the different coatings is determined by an experimental evaluation of their coefficient of restitution. This energy requirement is assumed to be fouling specific. Empirical defouling functions are presented. They correlate the defouling effect for both particle materials under various impact conditions. The empirical correlations are developed into a simulation procedure to predict particle impact erosion and energy dissipation of coated surfaces in numerical indentation simulations.
\end{abstract}

Keywords: aircraft engine defouling, $\mathrm{CO}_{2}$ dry-ice blasting, $\mathrm{HSC}$ experiment, solid particle restitution

\section{INTRODUCTION}

Aircraft engine defouling is a current topic of research for commercial aircraft operators. Compressor fouling is caused by in-service ingestion and deposition of various types of dispersed solids and fluids. This leads to decreased engine efficiency and power output as well as higher fuel consumption, pollutant emission and increased operational cost. To counteract this, a number of aircraft engine defouling systems have been developed in recent decades. These are mostly based on solid (e.g. coal-dust, nut-shells) or liquid (e.g. water droplets, solvents) particle injection into the engine core while the engine is dry cranked.

KURZ and BRUN [1] gave a comprehensive experimental and theoretical overview of the principles of fouling. SYVERUD et al. [2] performed salt water deterioration tests on a jet engine test-rig. MEHER-HOMJI and BROMLEY [3] described engine fouling as well as different cleaning methods and SYVERUD and BAKKEN [4] reported significant outcomes from online water-wash tests. The current research at Hochschule Darmstadt and Dublin Institute of Technology in cooperation with Lufthansa Technik AG is a cleaning system based on pressurized air carrying dispersed $\mathrm{CO}_{2}$ dry-ice particles as cleaning medium into the dry cranked core engine. The particles clean the compressor blades by erosive wear. The basic principles of the system are described in [5]. Further details of the system including particle laden in-engine flow investigations are described by the authors in [6].

\section{STATE OF THE ART}

One of the key tasks of the above mentioned research is the numerical simulation of the new engine wash process with $\mathrm{CO}_{2}$ dry-ice. Such a simulation must incorporate an appropriate erosion prediction formulation. The wash process is to be simulated using the commercial numerical code ANSYS CFX, which incorporates the turbomachinery specific erosion 
models from FINNIE [7] and GRANT and TABAKOFF [8]. FINNIE [7] analyses the basic principles of ductile and solid target material erosion and gives basic relations between erosion rate, particle impact velocity and impact angle. GRANT and TABAKOFF [8] developed a full theoretical approach to predict particle trajectories and rebounding behavior, as well as erosive action of the particles investigated within the simulated turbomachinery. Neither model is designed to predict coating erosion.

\subsection{General erosion modeling}

The literature contains a number of semi-empirical erosion models. They were mostly developed for particular applications and can be described as follows, where $E R$ is the predicted amount of erosion:

$$
E R=\prod_{i}\left(K_{i}\right) \cdot v_{P}^{n} \cdot d_{P}^{m} \cdot F(\alpha) \cdot F(\psi)
$$

Typically, such models include a range of material-dependent factors (here $K_{\mathrm{i}}$ ), and power-law expressions (parameters $n$ and $m$ ) for particle velocity $v_{\mathrm{P}}$ and size $d_{\mathrm{P}}$. Some models also include functional relations ' $F$ ' of impact angle ' $\alpha$ ' and material properties (here ' $\psi$ '). One such model is the Tulsa erosion model $[9,10]$. It was developed to predict erosion by dispersed particles in oil pipelines. A comparable but more general model is that of OKA et al. [11, 12], which can be adapted to various situations with experimental calibration. Another approach is the microscale dynamic modelling described by LI et al. in [13, 14], which accounts for the mechanical properties of the material by direct simulation of the material matrix. The method is computationally expensive for large-scale simulations.

\subsection{Coating erosion}

The erosion of coatings without substrate penetration has been studied by a number of researchers. A selection of the most relevant studies to this work is listed in Table 1 and these are discussed below.

KIM et al. [15] reported increasing erosion rates with increasing coating hardness. ZHANG and DONG [16], DJUROVIC et al. [17] and LI et al. [18] determined particle impact velocity and diameter to be the most important variables influencing the erosion rate. Particle impact

Table 1: Overview of a range of experimental investigations from literature.

\begin{tabular}{lllll}
\hline Study & Coating & Particles & $d_{P}$-range $[\mu m]$ & $v_{P}$-range $[\mathrm{m} / \mathrm{s}]$ \\
\hline$[15]$ & WC-Ni & $\mathrm{Al}_{2} \mathrm{O}_{3}$ & 50 & 30 \\
{$[16]$} & metalic-ceramic-composite & catalyst & $60 \ldots 120$ & $50 \ldots 250$ \\
{$[17]$} & organic & wheat starch & $400,600,1100$ & $140 \ldots 250$ \\
{$[18]$} & paint & ceramics & $300 \ldots 900$ & $47 \ldots 116$ \\
{$[19]$} & TBC ceramics & $\mathrm{Al}_{2} \mathrm{O}_{3}$ & $16.7,126$ & 60,104 \\
{$[20]$} & paint & $\mathrm{CO}_{2}$ dry-ice & 500 & $155 \ldots 241$ \\
{$[21]$} & paint & steel, Al, polyacetat & 2000 & $35 \ldots 200$ \\
{$[22]$} & paint and chrome & Glass & 255 & 73,110 \\
{$[23]$} & polyamide \& polyurethane & Steel & 890 & 55 \\
\hline
\end{tabular}


velocity was stated to be the most important erosion factor by CERNUSCHI et al. [19] and by WESTON et al. [20]. The latter also found target temperature to influence the erosive behavior. ZOUARI and TOURATIER [21] reported detailed penetration, buckling and delamination mechanism investigations of paint coatings upon indentation. SHIPWAY et al. [22] investigated cracking, fracture and bending processes for paint and chrome coatings. PAPINI and SPELT [23] investigated elastic-plastic mechanisms in polyamide and polyurethane coatings upon single particle impact.

\subsection{Dynamic indentation testing}

In order to determine the behaviour of fouling material under erosion during engine cleaning, this study uses an experimental single-particle indention process. Several other researchers have taken a comparable approach and their work is summarised below.

HUTCHINGS et al. [24] and later in Ref. [25] investigated the restitution properties of single particles of steel, quartz and tungsten carbide (WC) impacting mild steel targets with a range of velocities and impact angles. The restitution behaviour of the particles was investigated experimentally. Crater volume and dissipated energy were found to be functions of impact angle and velocity, and similarity between craters from particles of different material was reported. A crater formation and energy consumption model was generated from this work and implemented in software [26].

SUNDARAJAN et al. called the above experimental technique 'Dynamic Indentation Testing' (DI) and performed comparable experiments with single steel [27] and WC [28] particles impacting ductile target materials. In Ref. [27] an energy balance based model was introduced to predict crater volume and particle rebound characteristics. The authors introduced dynamic hardness as a material property. They also defined a range of requirements to ensure validity of the DI method, including quasi-static impact behaviour, negligible stress-wave energy losses, particle hardness greater than target hardness and the ability to add together losses calculated from normal and tangential forces.

BARNOCKY and DAVIS [29] and DAVIS et al. [30] experimentally investigated rebound characteristics for single particles made from plastic and metal impacting quartz targets coated with viscous fluids. They discussed the coefficient of restitution for single particles as a function of Stokes number, coating thickness and substrate roughness. They found the viscous layer responsible for lower restitution coefficients due to higher energy dissipation in certain Stokes number ranges.

PAPINI and SPELT [31] investigated glass bead impacts against alkyd coated steel substrates to assess coating removal. They measured energy losses and restitution behavior of the impacting particles and developed an energy-based model to predict coating removal. They found coating removal to be independent of tangential forces and reported the shape of indentation changing at increased impact angles. Furthermore, they found that the coefficient of restitution can be used to determine energy dissipation over a range of normal impact velocities up to a certain maximum. Coating thickness was found to be negligible in indentation surface estimations. The onset of erosion was related to full penetration of the coating.

WALL et al. [32] investigated the rebound behavior of ammonium fluoride particles impinging upon a range of target materials to obtain data for particle capture and energy dissipation. They introduced an energy-based impact formulation and found the coefficient of restitution to be dependent on particle diameter. This dependence is reported to be a function of impingement velocity. Furthermore they reported measurable differences in particle 
restitution coefficients depending on the target material. The differences are reported to be measurable only over a certain velocity range.

GONDRET et al. $[33,34]$ gave an overview of single-particle restitution behavior for various particles rebounding from various target materials in a range of fluids without indentation or erosion. They reported the fluid playing a non-negligible role in coefficient of restitution determination. Normalized coefficient of restitution is reported to be a function of Stokes number. This relation is independent of the fluid-particle-wall combinations considered.

HUSSAINOVA and KLEIS reported specific crater-formation energy as a material constant representing its dynamic hardness, which can be derived from single particle impact experiments and energy-based theory [35, 36]. In further studies [37, 38], the energy-based description was modified by momentum-based impact formulations allowing the calculation of kinetic energy losses. These can be described by restitution and friction coefficients based on the sliding and rolling regime of the impacting particle upon wall contact.

\section{MODEL DESCRIPTION}

\subsection{Theoretical formulation}

The model presented here calculates particle energy dissipation during fouling erosion. Basically following GONDRET et al. [33, 34], the process is assumed to be dependent on the Stokes number St, which incorporates the influence of particle size (diameter $d$ ), mass $\rho$, and velocity $v$ :

$$
S t=\left(\frac{1}{9}\right) \cdot \frac{\rho_{P} \cdot v_{P} \cdot d_{P}}{\eta_{F}} .
$$

Equation (3) describes the dissipated portion of impact velocity $\delta v_{P}^{\{f o u\}}$ required for a particle of a particular material (subscript part) to produce an indentation in a particular fouling layer (superscript fou). This is determined using the difference between experimentally measured coefficients of restitution for impacts on unfouled $\varepsilon$ and fouled $\varepsilon^{\{f o u\}}$ targets:

$$
\delta v_{P}^{\{f o u\}}=\left[\varepsilon(S t)-\varepsilon^{\{f o u\}}(S t)\right] \cdot v_{P}^{\{p a r t\}}:=\delta \varepsilon^{\{f o u\}}(S t) \cdot v_{P}^{\{p a r t\}} .
$$

Here, $\delta \varepsilon^{\{f o u\}}$ is assumed to be a fouling-specific property. It has previously been established (see e.g. [29-31]) that fouling must be the only factor which causes these coefficient of restitution differences, and since $\mathrm{CO}_{2}$ dry-ice particles tend to disintegrate on impact (see for example Ref. [6]) the required data for eqn. (3) must be obtained using a reference particle material (superscript ref), which does not plastically deform in the given range of Stokes numbers. Considering this, the dissipated portion of specific energy upon fouling indentation $\delta$ e can be described:

$$
\delta e^{\{p a r t, f o u, a\}}=\frac{\rho_{\text {ref }}}{2} \cdot\left[\delta v_{P}^{\{f o u\}}\right]^{2} \cdot\left[\frac{d_{I M P}^{\{p a r t, f o u, a\}}(S t)}{d_{I M P}^{\{r e f, f o u, 0\}}(S t)}\right]^{2} .
$$

In this equation, the additional superfix $\alpha$ represents the impact angle (measured normal to the wall). The quotient on the right hand side of eqn. (4) consists of the area defouled by the particle material regarded (part) related to the area defouled by the non-disintegrating reference 
particle material (ref) impacting in the normal direction. Data from the normal impacts is used for determination of the dissipated portion of impact velocity (eqn. (3)).

Both areas are calculated by means of the experimentally correlated (correlation coefficients $K_{1}$ and $K_{2}$ ) indentation diameter $d_{I M P}$

$$
d_{I M P}^{\{\text {part }, \text { fou }, a\}}=\left\{\begin{array}{ll}
0 & S t \leq S t_{\text {crit }} \\
K_{2}^{\{\text {part }, \text { fou }, a\}} \cdot \ln (S t)+K_{1}^{\{p a r t, f o u, a\}} & S t>S t_{\text {crit }}
\end{array} .\right.
$$

The onset of erosion for the particle-fouling combination under consideration is described by the critical Stokes number $S t_{\text {crit }}$.

The quotient described from eqn. (4) is used to scale the dissipated portion of energy consumed by the defouling from reference material ( $r e f)$ values to actual material (part) values. The defouled area $A_{I M P}$ from one single particle impact is consequently calculated:

$$
A_{I M P}^{\{\text {part }, f o u, a\}}=\frac{\pi}{4}\left(d_{I M P}^{\{\text {part }, f o u, a\}}(S t)\right)^{2}
$$

\subsection{Basic experiment}

The experimental setup for the single-particle indentation tests is displayed in Fig. 1. It consists of a gas gun, which accelerates a particle carriage (a) by compressed air (b) through a cylinder (c). The carriage is stopped by an end-of-stroke damper (d) and the particle is projected towards the target plate placed above (e). Its angle can be varied. Particle size and velocity is measured by means of a high-speed camera (HSC) (f) before and after impact. The data is processed using Matlab post-processors developed by the authors [6]. For fouled targets, the indentation is measured by a before-after comparison of impact area photographs. These are recorded by means of the second camera $(\mathrm{g})$.

\section{RESULTS}

The experimental outcomes discussed in this study deal with an indicator coating (PTFE), which is used in $\mathrm{CO}_{2}$ dry-ice aircraft engine blasting experiments at Hochschule Darmstadt to predict particle blade contact regions. In addition, layers of actual fouling (FOU) from low-pressure compressor blades of commercial aircraft engines are investigated. These are taken directly from service.

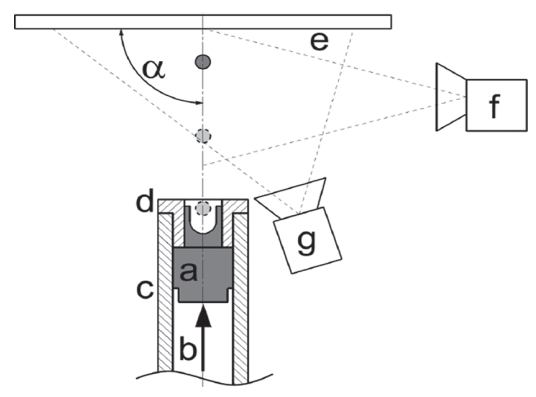

Figure 1: Schematic diagram of the experimental setup. 


\subsection{Experiment}

Figure 2 shows the results from the reference material restitution data (POM spheres) for PTFE (left, red x-markers) and actual fouling (right, red +-markers) in comparison to unfouled target restitution (blue o-markers in both graphs). A difference can be seen between PTFE and unfouled results in the range of Stokes number from $1 \times 10^{3}$ to $200 \times 10^{3}$. In contrast, the particle restitution from actual fouling is similar to the unfouled target for low Stokes numbers, but for Stokes numbers from $200 \times 10^{3}$ to $400 \times 10^{3}$ there is a difference measurable. For higher Stokes numbers the difference becomes indistinct (comparable with findings from $[31,32])$. The trends described are correlated in the range of Stokes numbers where a difference is visible and the results are incorporated into eqn. (3).

Diameters of the defouling indentations for PTFE are displayed in Fig. 3. Impact diameters are normalized by the maximum indentation diameter encountered within the experiments presented here. Only normal impact results are displayed. The left-hand graph of Fig. 3 shows normalised indentation diameter as a function of Stokes number for PTFE fouling and POM particles. It represents the combination of ideal fouling vs. ideal spherical, non- disintegrative particle. The experimental data (blue o-markers) are correlated logarithmically with $\mathrm{R}^{2}>0.90$.


Figure 2: Coefficients of restitution for POM (ref) particles impacting clean target (blue markers), PTFE (left) and actual fouling (right).
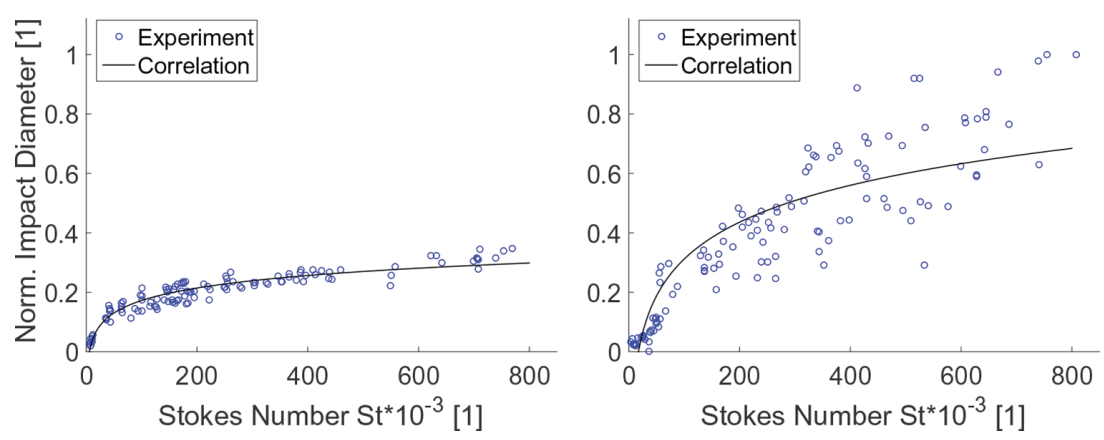

Figure 3: Normalized impact diameter for PTFE from POM (left) and $\mathrm{CO}_{2}$ (right) particle indentations. 

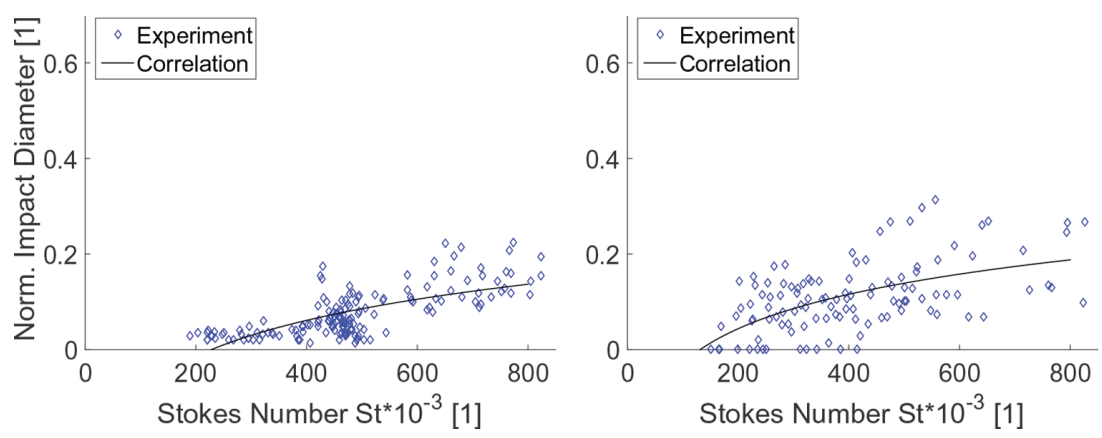

Figure 4: Normalized impact diameter for actual fouling from POM (left) and $\mathrm{CO}_{2}$ (right) particle indentations.

The right-hand graph of Fig. 3 shows the same PTFE layer indented by $\mathrm{CO}_{2}$ dry-ice particles. The global trends are comparable to these for the POM particles. A wider scattering at higher Stokes numbers is encountered. The logarithmic fit is determined with $R^{2}>0.75$.

Investigation of the actual fouling reveals the trends shown in Fig. 4. The POM indentations (Fig. 4, left) have a greater scatter than their PTFE impacts (Fig. 3, left). This can be explained by the inhomogenous and amorphous topology of the actual fouling. The coefficient of determination is $R^{2}=0.50$. Figure 4, right, contains actual fouling indentation data from $\mathrm{CO}_{2}$ dry-ice particles. The scatter becomes greater due to the $\mathrm{CO}_{2}$ dry-ice morphology explained above. The trend fits the experimental data with $R^{2}=0.36$. The critical Stokes number indicating the onset of defouling, derived from the experiments, is comparable: for POM $\mathrm{St}_{c r i t}=188 \times 10^{3}$ and for $\mathrm{CO}_{2}$ dry-ice $\mathrm{St}_{\text {crit }}=169 \times 10^{3}$. Figure 2, right, confirms this indication as the first differences for the restitution coefficients from actual fouled targets can be observed at $\mathrm{St}=190 \times 10^{3}$.

\subsection{Simulation}

Utilizing all datasets generated in this work in eqns. (4-6) the simulation procedure is obtained. All computations presented are made with a Matlab code developed at Hochschule Darmstadt.

Figure 5 shows the simulation results for the normalized defouled area (according eqn. (6)) from $\mathrm{CO}_{2}$ dry-ice particle impacts. The impact angle is varied within the range investigated experimentally ( 0 and 60). It can be seen that the impact angle plays a minor role for PTFE defouling in the given range of Stokes numbers. The PTFE behaviour is comparable to that of alkyd coatings reported in Ref. [31]. In the right-hand graph of Fig. 5 the proportion of particle kinetic energy required to penetrate and remove the predicted amount of fouling is shown. It can be seen that this energy is dependent on the impact angle. The measurable differences in the coefficient of restitution (see Fig. 2, left) disappear for Stokes numbers $\mathrm{St}>200 \times 10^{3}$. It can therefore be concluded that particle energy lost to coating removal is negligible in terms of the particles overall energy.

Results comparable to those discussed above are shown in Fig. 6 for the actual fouling simulations. The onset of defouling is found at higher Stokes numbers compared to PTFE (Fig. 5 and Fig. 6, left). The defouling results are dependent on the particle impact angle. The results for 60 particle impacts show negligible indentations even at high Stokes numbers. This behavior is comparable to what is reported for urethane and paint coatings in [17] and 

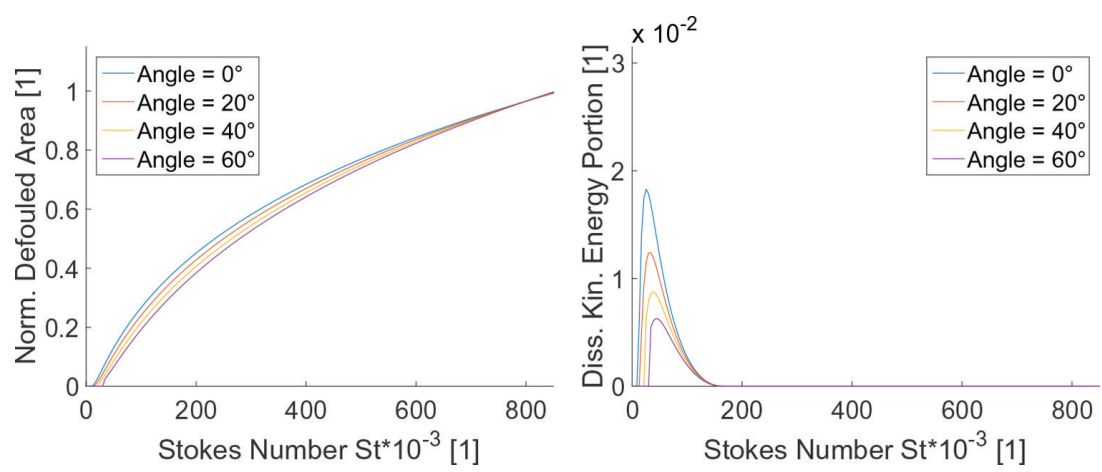

Figure 5: Normalized defouled area for PTFE fouling (left) and dissipated kinetic energy (right) from $\mathrm{CO}_{2}$ particle impact simulations.
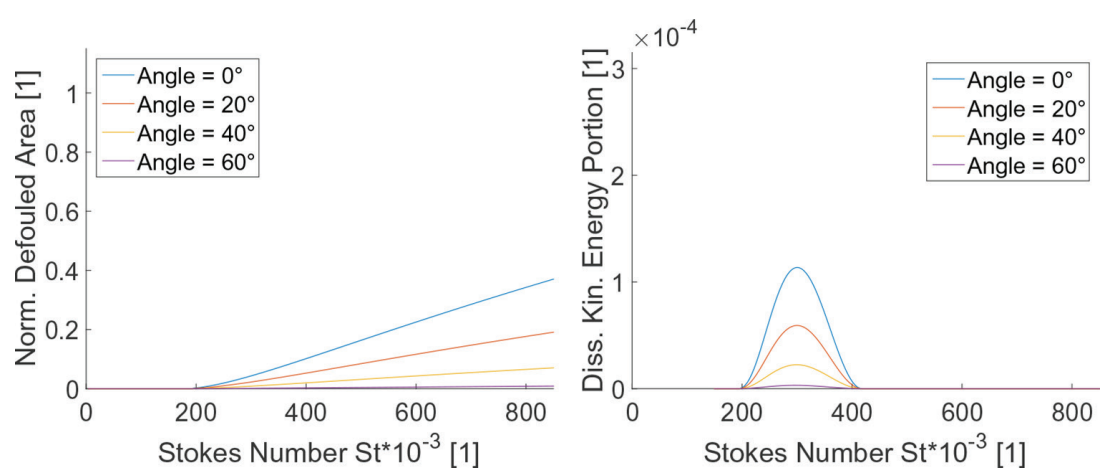

Figure 6: Normalized defouled area for actual fouling (left) and dissipated kinetic energy (right) from $\mathrm{CO}_{2}$ particle impact simulations.

TBC coatings in [19]. The energy proportion dissipated during defouling is measurable only in the range of Stokes numbers discussed above (Fig. 6, right). The predicted proportion of energy is negligible in an overall energy balance of the impacting particle.

\section{CONCLUSION AND OUTLOOK}

An experimentally based simulation procedure to predict fouling layer erosion by single particle impacts is presented. It predicts the proportion of impacting particle energy dissipated during fouling removal by means of a comparison of coefficients of restitution. This technique is comparable to what is reported elsewhere $[27,28]$ as the dynamic indentation testing.

A defouling function, correlated from indentation experiments for various fouling-particle combinations, describing the actual defouling action of single particle impacts is presented. It is used to scale the proportion of energy dissipated from a reference particle material value (POM) to the particle material actually being used (e.g. $\mathrm{CO}_{2}$ dry-ice). The simulation procedure will be incorporated into Ansys CFX for use of $\mathrm{CO}_{2}$ dry-ice blasting simulations in commercial aircraft engines. The results from these simulations will be reported in a future communication. 
Future work will encompass a systematic enlargement of the statistical database for the model presented here. Experiments for measurement of actually fouled high-pressure compressor blades (taken from service) as well as investigations of compressor blades fouled with salt layers are planned. Tests with water ice particles instead of $\mathrm{CO}_{2}$ dry-ice will also be performed. Furthermore, it is planned to use the model in the context of more general $\mathrm{CO}_{2}$ dry-ice blasting investigations. It is intended to apply this model in another research project at Hochschule Darmstadt involving fouling layers typically found in tyre production moulds.

\section{REFERENCES}

[1] Kurz, R. \& Brun, K., Fouling mechanisms in axial compressors. Journal of Engineering for Gas Turbines and Power, 134, pp. 1-9, 2012. https://doi.org/10.1115/1.4004403

[2] Syverud, E., Brekke, O. \& Bakken, L.E., Axial compressor deterioration caused by saltwater ingestion. Proceedings of GT2005, ASME Turbo Expo, Reno-Tahoe, Nevada, USA, pp. 1-11, 2005.

[3] Meher-Homji, C. \& Bromley, A., Gas turbine axial compressor fouling and washing. Proceedings of the 33rd Turbomachinery Symposium, pp. 163-191, 2004.

[4] Syverud, E. \& Bakken, L.E., Online water wash tests of ge j85-13. Proceedings of GT2005, ASME Turbo Expo, Reno-Tahoe, Nevada, USA, pp. 1-9, 2005.

[5] Giljohann, S., Braeutigam, K., Kuhn, S., Annasiri, S. \& Russ, G., Investigations into the on-wing cleaning of commercial jet engines with co2 dry ice blasting. Deutscher Luft- und Raumfahrkongress, Berlin, Germany, pp. 1-9, 2012.

[6] Rudek, A., Russ, G. \& Duignan, B., Particle laden flow investigations in special purpose dry-ice blasting applications. International Journal of Computational Methods and Experimental Measurement, 4, pp. 393-402, 2016.

https://doi.org/10.2495/cmem-v4-n4-393-402

[7] Finnie, I., Erosion of surfaces by solic particles. Wear, 3, pp. 87-103, 1960. https://doi.org/10.1016/0043-1648(60)90055-7

[8] Grant, G. \& Tabakoff, W., Erosion prediction in turbomachinery resulting from environmental solid particles. Journal of Aircraft, 12, pp. 471-478, 1975. https://doi.org/10.2514/3.59826

[9] Chen, X., McLaury, B.S. \& Shirazi, S.A., Numerical and experimental investigation of the relative erosion severity between plugged tees and elbows in dilute gas/solid twophase flow. Wear, 261, pp. 715-729, 2006.

https://doi.org/10.1016/j.wear.2006.01.022

[10] Zhang, Y., McLaury, B.S., Shirzai, S.A. \& Russell, R.D., Simulations of particle-wallturbulence interactions, particle motion and erosion in with a commercial CFD code. Proceedings of FEDSM2006, 2006 ASME Joint US - European Fluids Engineering Summer Meeting, Miami, Florida, USA, pp. 1-16, 2006.

[11] Oka, Y.I., Okamura, K. \& Yoshida, T., Practical estimation of erosion damage caused by solid particle impact part 1: Effects of impact parameters on a predictive equation. Wear, 259, pp. 95-101, 2005. https://doi.org/10.1016/j.wear.2005.01.039

[12] Oka, Y. \& Yoshida, T., Practical estimation of erosion damage caused by solid particle impact part 2: Mechanical properties of materials directly associated with erosion damage. Wear, 259, pp. 102-109, 2005.

https://doi.org/10.1016/j.wear.2005.01.040 
[13] Li, D., Elalem, K., Anderson, M. \& Chiovelli, S., A microscale dynamical model for wear simulation. Wear, 225-229, pp. 380-386, 1999.

https://doi.org/10.1016/s0043-1648(98)00368-8

[14] Chen, Q. \& Li, D., Computer simulation of solid particle erosion. Wear, 254, pp. 203-210, 2003.

https://doi.org/10.1016/s0043-1648(03)00006-1

[15] Kim, J.H., Joob, H.G. \& Lee, K.Y., Simulation of solid particle erosion in WC-NI coated wall using CFD. Journal of Materials Processing Technology, 224, pp. 240-245. https://doi.org/10.1016/j.jmatprotec.2015.01.022

[16] Zhang, H. \& Dong, X., Finite element analysis of multiple solid particles erosion in cermet coating. Surface and Coatings Technology, 262, pp. 184-190, 2015. https://doi.org/10.1016/j.surfcoat.2014.12.040

[17] Djurovic, B., Jean, E., Papini, M., Tangestanian, P. \& Spelt, J.K., Coating removal from fiber-composites and aluminum using starch media blasting. Wear, 224, pp. 22-37, 1999. https://doi.org/10.1016/s0043-1648(98)00308-1

[18] Li, M.Z., Liu, W.W., Qing, X.C., Yu, Y., Liu, L.H., Tang, Z.J., Wang, H.J., Dong, Y.Z. \& Zhang, H.C., Feasibility study of a new approach to removal of paint coatings in remanufacturing. Journal of Materials Processing Technology, 234, pp. 102-112, 2016. https://doi.org/10.1016/j.jmatprotec.2016.03.014

[19] Cernuschi, F., Guardamagna, C., Capelli, S., Lorenzoni, L., Mack, D.E. \& Moscatelli, A., Solid particle erosion of standard and advanced thermal barrier coatings. Wear, 348-349, pp. 43-51, 2016. https://doi.org/10.1016/j.wear.2015.10.021

[20] Weston, D.P., Shipway, P.H. \& Harris, S.J., Coating removal from an industrial polypropylene blend by cryogenic blasting: The development of substrate damage. Wear, 258, pp. 392-401, 2005. https://doi.org/10.1016/j.wear.2004.01.021

[21] Zouari, B. \& Touratier, M., Simulation of organic coating removal by particle impact. Wear, 253, pp. 488-497, 2002. https://doi.org/10.1016/s0043-1648(02)00141-2

[22] Shipway, P.H., Bromley, J.P.D. \& Weston, D.P., Removal of coatings from polymer substrates by solid particle blasting to enhance reuse or recycling. Wear, 263, pp. 309-317, 2007. https://doi.org/10.1016/j.wear.2006.11.032

[23] Papini, M. \& Spelt, J.K., The plowing erosion of organic coatings by spherical particles. Wear, 222, pp. 38-48, 1998. https://doi.org/10.1016/s0043-1648(98)00274-9

[24] Hutchings, I.M. \& Winter, R.E., The erosion of ductile metals by spherical particles. Journal of Applied Physics, 8, pp. 8-17, 1975. https://doi.org/10.1088/0022-3727/8/1/010

[25] Hutchings, I.M., Winter, R.E. \& Field, J.E., Solid particle erosion of metals: The removal of surface material by spherical projectiles. Proceedings of the Royal Society of London Series A, Mathematical and Physical Sciences, 348, pp. 379-392, 1976.

[26] Hutchings, I., Further studies of the oblique impact of a hard sphere against a ductile solid. International Journal of Mechanical Sciences, 23, pp. 639-646, 1981. https://doi.org/10.1016/0020-7403(81)90018-7 
[27] Sundararajan, G. \& Shewmon, P.G., The oblique impact of a hard ball against ductile, semi-infinite target materials experiment and analysis. International Journal of Impact Engineering, 6, pp. 3-22, 1987.

https://doi.org/10.1016/0734-743x(87)90003-0

[28] Tirupataiah, Y. \& Sundararajan, G., A dynamic indentation technique for the characterization of the high strain rate plastic flow behaviour of ductile metals and alloys. Journal of Mechanics and Physics of Solids, 39, pp. 243-271, 1991. https://doi.org/10.1016/0022-5096(91)90005-9

[29] Barnocky, G. \& Davis, R.H., Elastohydrodynamic collision and rebound of spheres: Experimental verification. Physics of Fluids, 31, pp. 1324-1329, 1988. https://doi.org/10.1063/1.866725

[30] Davis, R.H., Rager, D.A. \& Good, B.T., Elastohydrodynamic rebound of spheres from coated surfaces. Journal of Fluid Mechanics, 468, pp. 107-119, 2002. https://doi.org/10.1017/s0022112002001489

[31] Papini, M. \& Spelt, J.K., Organic coating removal by particle impact. Wear, 213, pp. 185-199, 1997. https://doi.org/10.1016/s0043-1648(97)00062-8

[32] Wall, S., John, W., Wang, H.C. \& Goren, S.L., Measurements of kinetic energy loss for particles impacting surfaces. Aerosol Science and Technology, 12, pp. 926-946, 1990. https://doi.org/10.1080/02786829008959404

[33] Gondret, P., Hallouin, E., Lance, M. \& Petit, L., Experiments on the motion of a solid sphere toward a wall: from viscous dissipation to elastohydrodynamic bouncing. Physics of Fluids, 11, pp. 2803-2805, 1999. https://doi.org/10.1063/1.870109

[34] Gondret, P., Lance, M. \& Petit, L., Bouncing motion of spherical particles in fluids. Physics of Fluids, 14, pp. 643-652, 2002. https://doi.org/10.1063/1.1427920

[35] Kleis, I. \& Hussainova, I., Investigation of particle wall impact process. Wear, 233-235, pp. 168-173, 1999. https://doi.org/10.1016/s0043-1648(99)00175-1

[36] Kleis, I. \& Kulu, P., Solid particle erosion. Springer, 2008.

[37] Hussainova, I., Schade, K.P. \& Tisler, S., Dynamic coefficients in impact mechanics. Proceedings of Estonian Academic and Scientific Engineering, 12, pp. 26-39, 2006.

[38] Hussainova, I. \& Schade, K.P., Correlation between solid particle erosion of cermets and particle impact dynamics. Tribology International, 41, pp. 323-330, 2008. https://doi.org/10.1016/j.triboint.2007.09.001 